\title{
Correlations and fluctuations of conserved charges from lattice QCD
}

\author{
Szabolcs Borsanyi ${ }^{a}$, Zoltan Fodor ${ }^{a, b, c}$, Sandor D. Katz ${ }^{a, c}$, Stefan Krieg ${ }^{a, b}$, Claudia \\ Ratti $^{* d}$, Kalman K. Szabo ${ }^{a}$ \\ ${ }^{a}$ Bergische Universität Wuppertal, D-42119 Wuppertal, Germany \\ ${ }^{b}$ IAS, Jülich Supercomputing Centre, Forschungszentrum Jülich, D-52425 Jülich, Germany \\ ${ }^{c}$ Institute for Theoretical Physics, Eötvös University, H-1117 Budapest, Hungary \\ ${ }^{d}$ Università degli Studi di Torino and INFN, Sezione di Torino, I-10125 Torino, Italy \\ E-mail: ratti@to.infn.it
}

\begin{abstract}
We review the latest results of the Wuppertal-Budapest lattice QCD collaboration on flavor diagonal and non-diagonal quark number susceptibilities with $2+1$ staggered quark flavors, in a temperature range between 125 and $400 \mathrm{MeV}$. A Symanzik improved gauge and a stout-link improved staggered fermion action is utilized; the light and strange quark masses are set to their physical values, with a ratio $m_{s} / m_{u, d}=28.15$. Lattices with $N_{t}=6,8,10,12,16$ are used. A continuum extrapolation of all observables under study is performed. All results are compared to the Hadron Resonance Gas model predictions: good agreement is found in the temperature region below the phase transition.
\end{abstract}

Sixth International Conference on Quarks and Nuclear Physics,

April 16-20, 2012

Ecole Polytechnique, Palaiseau, Paris

\footnotetext{
${ }^{*}$ Speaker.
} 


\section{Introduction}

The study of the QCD phase diagram and thermodynamics is particularly appealing in recent days because the deconfined phase of QCD can be produced in the laboratory, in the ultrarelativistic heavy ion collision experiments at CERN SPS, RHIC at Brookhaven National Laboratory, ALICE at the LHC and the future FAIR at the GSI. It was shown that the transition from the hadronic, confined system to the phase dominated by colored degrees of freedom is merely an analytic crossover at small densities [1]. A lot of effort is invested, both theoretically and experimentally, in order to find observables which would unambiguously signal the QCD phase transition. Correlations and fluctuations of conserved charges have been proposed long ago to this purpose [2, 3]. These quantum numbers have a very different value in a confined and deconfined system; therefore, measuring them in the laboratory allows to distinguish between the two phases.

Fluctuations of conserved charges can be obtained as linear combinations of diagonal and non-diagonal quark number susceptibilities, which can be calculated on the lattice at zero chemical potential $[4,5]$. These observables can give us an insight on the nature of the matter under study $[4,6]$. Diagonal susceptibilities measure the response of the quark number density to changes in the chemical potential, and show a rapid rise in the vicinity of the phase transition. Non-diagonal susceptibilities give us information about the correlation between different flavors. They are supposed to vanish in a non-interacting quark-gluon plasma (QGP). It was shown in Ref. [7] that correlations between different flavors are nonzero in perturbative QCD at large temperatures due to the presence of flavor-mixing diagrams: a quantitative analysis of this observable allows one to get an insight on the presence of bound states in the QGP [8]. Another observable which was proposed to this purpose, and which can be obtained from a combination of diagonal and non-diagonal quark number susceptibilities, is the baryon-strangeness correlator [9]. Quark number susceptibilities also allow to expand thermodynamic quantities in Taylor series (which is a truncation of the full multiparameter reweighting method $[10,11])$ around $\mu=0$ [12].

In the present contribution we show the results of our collaboration on some of these observables, with 2+1 staggered quark flavors, in a temperature regime between 125 and $400 \mathrm{MeV}$ [13]. The light and strange quark masses are set to their physical values, with a ratio $m_{s} / m_{u, d}=28.15$. Lattices with $N_{t}=6,8,10,12,16$ are used. Continuum extrapolations are performed for all observables under study. We compare our results to the predictions of the HRG model with resonances up to $2.5 \mathrm{GeV}$ mass at small temperatures, and of the Hard Thermal Loop (HTL) resummation scheme at large temperatures, when available. All details can be found in [13].

\section{Observables under study}

The baryon number $B$, strangeness $S$ and electric charge $Q$ fluctuations can be obtained, at vanishing chemical potentials, from the QCD partition function. The relationships between the quark chemical potentials and those of the conserved charges are:

$$
\mu_{u}=\frac{1}{3} \mu_{B}+\frac{2}{3} \mu_{Q} ; \quad \mu_{d}=\frac{1}{3} \mu_{B}-\frac{1}{3} \mu_{Q} ; \quad \mu_{s}=\frac{1}{3} \mu_{B}-\frac{1}{3} \mu_{Q}-\mu_{S} .
$$

Starting from the QCD pressure,

$$
\frac{p}{T^{4}}=\frac{1}{V T^{3}} \ln Z\left(V, T, \mu_{B}, \mu_{S}, \mu_{Q}\right)
$$


we can define the moments of charge fluctuations as follows:

$$
\chi_{\text {lmn }}^{B S Q}=\frac{\partial^{l+m+n} p / T^{4}}{\partial\left(\mu_{B} / T\right)^{l} \partial\left(\mu_{S} / T\right)^{m} \partial\left(\mu_{Q} / T\right)^{n}} .
$$

In the present paper we will concentrate on the quadratic fluctuations and on the correlators among different charges or quark flavors. Given the relationships between chemical potentials (2.1) the diagonal susceptibilities of the conserved charges can be obtained from quark number susceptibilities in the following way:

$$
\begin{aligned}
\chi_{2}^{B} & =\frac{1}{9}\left[\chi_{2}^{u}+\chi_{2}^{d}+\chi_{2}^{s}+2 \chi_{11}^{u s}+2 \chi_{11}^{d s}+2 \chi_{11}^{u d}\right], \\
\chi_{2}^{Q} & =\frac{1}{9}\left[4 \chi_{2}^{u}+\chi_{2}^{d}+\chi_{2}^{s}-4 \chi_{11}^{u s}+2 \chi_{11}^{d s}-4 \chi_{11}^{u d}\right], \\
\chi_{2}^{S} & =\chi_{2}^{s} .
\end{aligned}
$$

The baryon-strangeness correlator, which was proposed in Ref. [9] as a diagnostic to understand the nature of the degrees of freedom in the QGP, is defined in the following way:

$$
C_{B S}=-3 \frac{\left\langle N_{B} N_{S}\right\rangle}{\left\langle N_{S}^{2}\right\rangle}=1+\frac{\chi_{11}^{u s}+\chi_{11}^{d s}}{\chi_{2}^{s}}
$$

\section{Results and conclusions}

The behavior of light and strange quark number susceptibilities as functions of the temperature is shown in the two panels of Fig. 1. The different symbols correspond to different values of $N_{t}$, from 6 to 16. The continuum extrapolation is performed through a parabolic fit in the variable $\left(1 / N_{t}\right)^{2}$, over five $N_{t}$ values from 6 to 16 . The band shows the spread of the results of other possible fits. Both observables show a rapid rise in a certain temperature range, and reach approximately $90 \%$ of the ideal gas value at large temperatures. However, the temperature around which the susceptibilities rise is approximately $15-20 \mathrm{MeV}$ larger for strange quarks than for light quarks. The pattern of temperature dependence is strongly related to the actual quark mass. The difference between the light and strange susceptibilities here with physical masses is more pronounced than in earlier works with not so light pions (see Ref. [14]).

The non-diagonal us susceptibility measures the degree of correlation between different flavors. This observable vanishes in the limit of an ideal, non-interacting QGP. We show our result in the left panel of Fig. 2. $\chi_{11}^{u s}$ is non-zero in the entire temperature range under study. It has a dip in the vicinity of the transition, where the correlation between $u$ and $s$ quarks turns out to be maximal. It agrees with the HRG model prediction in the hadronic phase. This correlation stays finite and large for a certain temperature range above the transition temperature $T_{c}[15,16,17]$. A quantitative comparison between lattice results and predictions for a purely partonic QGP state can give us information about the probability of bound states survival above $T_{C}$ [8]. The baryon-strangeness correlator $C_{B S}$ defined in Eq. (2.5) is supposed to be equal to one for a non-interacting QGP, while it is generally smaller than one in a hadronic system. We show our result for this observable in the right panel of Fig. 2. For small temperatures it agrees with the HRG model result, and it shows a rapid rise across the phase transition. It reaches the ideal gas limit much faster than the other 

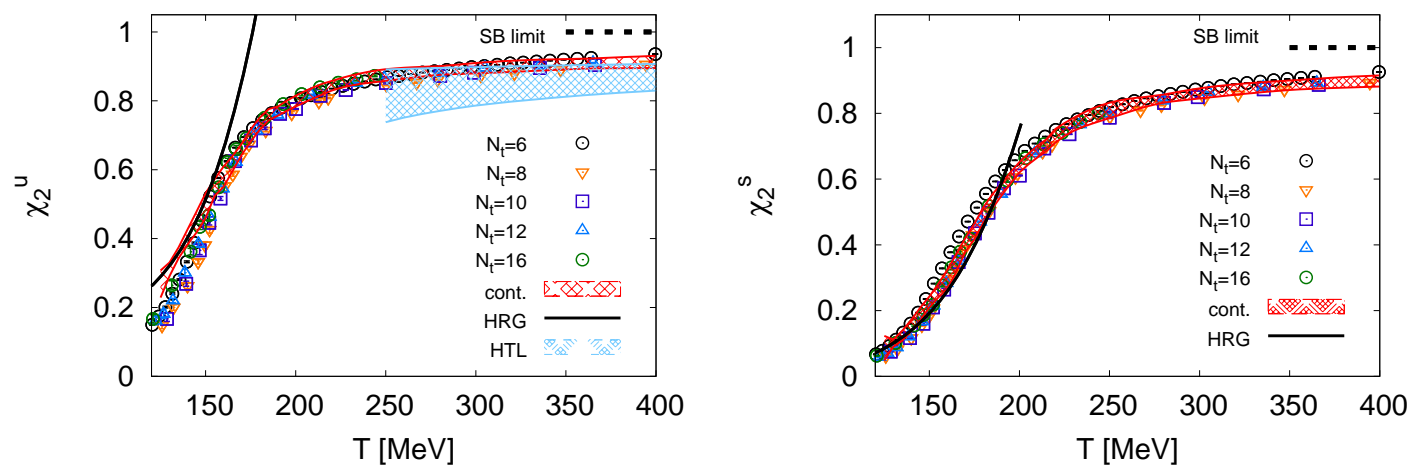

Figure 1: Left panel: diagonal light quark susceptibility as a function of the temperature. Right panel: diagonal strange quark susceptibility as a function of the temperature. In both panels, the different symbols correspond to different $N_{t}$ values. The red band is the continuum extrapolation. The black curve is the HRG model prediction for these observables. The dashed line shows the ideal gas limit. The light blue band in the left panel is the HTL prediction taken from Ref. [7].
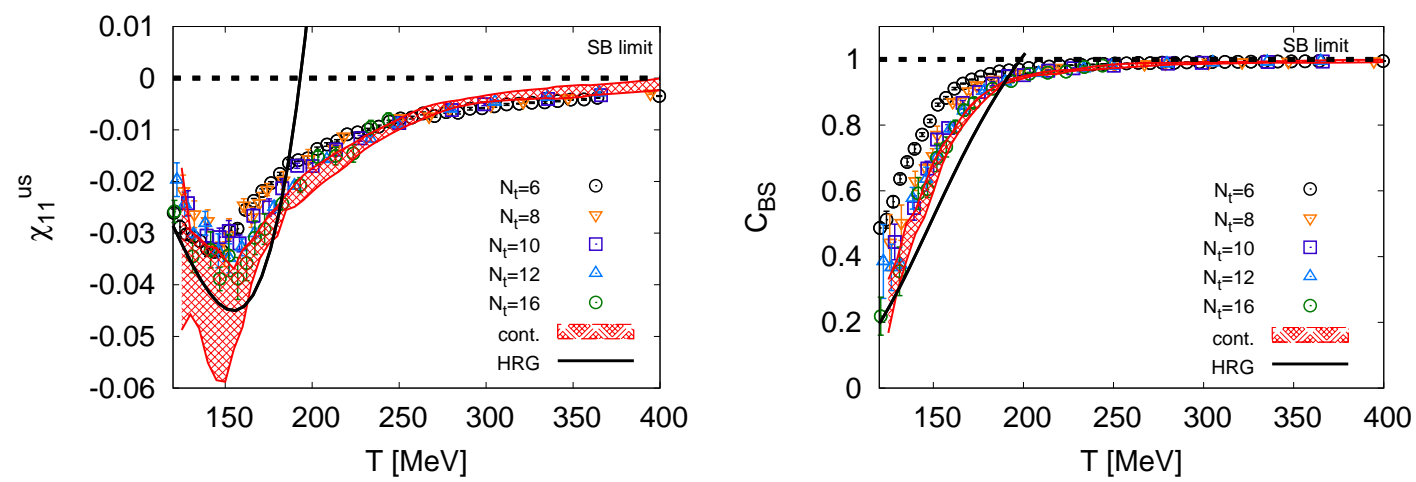

Figure 2: Left panel: Non-diagonal u-s correlator as a function of the temperature. Right panel: Baryonstrangeness correlator as a function of the temperature. In both panels, the different symbols correspond to different $N_{t}$ values, the red band is the continuum extrapolation and the black, solid curve is the HRG model result. The ideal gas limit is shown by the black, dashed line.

observables under study, yet there is a window of about $100 \mathrm{MeV}$ above $T_{c}$, where its value is still smaller than one. Quadratic baryon number and electric charge fluctuations can be obtained from the above partonic susceptibilities through Eqs. (2.4). We show our results for these observables in Fig. 3. In the low-temperature, hadronic phase we have a very good agreement with the HRG model predictions. In the vicinity of the phase transition, these quantities all show a rapid rise with temperature. At large temperature they reach approximately $90 \%$ of their ideal gas values.

\section{Acknowledgments}

Computations were carried out at the universities Wuppertal and Budapest on GPU clusters, on the Wuppertal QPACE machine and at Forschungszentrum Juelich. This work is supported in part by the Deutsche Forschungsgemeinschaft grants FO 502/2 and SFB- TR 55 and by the EU (FP7/2007-2013)/ERC no. 208740. The work of C. R. is supported by funds provided by the Italian Ministry of Education, Universities and Research under the Firb Research Grant RBFR0814TT. 

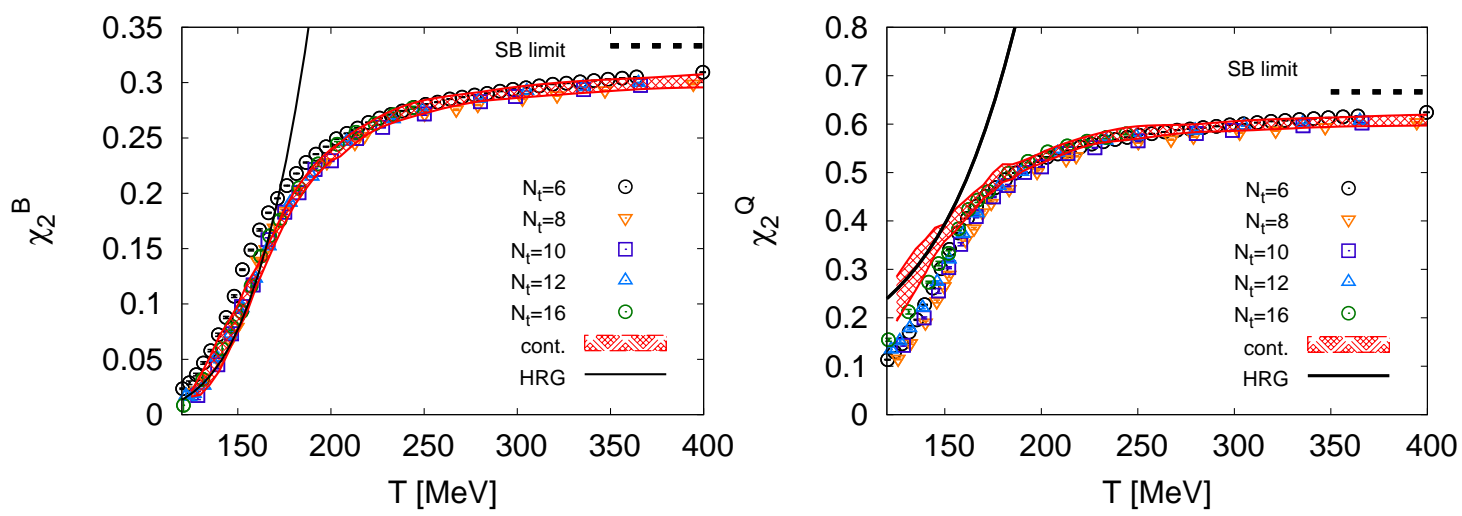

Figure 3: Left panel: quadratic fluctuation of baryon number as a function of the temperature. Right panel: electric charge susceptibility as a function of the temperature. In both panels, the different dots correspond to different $N_{t}$ values. The red band is the continuum extrapolation. The black curve is the HRG model prediction for these observables. The dashed line shows the ideal gas limit.

\section{References}

[1] Y. Aoki, G. Endrodi, Z. Fodor, S. D. Katz and K. K. Szabo, Nature 443, 675 (2006)

[2] S. Jeon and V. Koch, Phys. Rev. Lett. 85, 2076 (2000)

[3] M. Asakawa, U. W. Heinz and B. Muller, Phys. Rev. Lett. 85, 2072 (2000)

[4] S. A. Gottlieb, W. Liu, D. Toussaint, R. L. Renken, R. L. Sugar, Phys. Rev. Lett. 59, 2247 (1987). Phys. Rev. D38, 2888-2896 (1988).

[5] R. V. Gavai, J. Potvin, S. Sanielevici, Phys. Rev. D40, 2743 (1989).

[6] L. D. McLerran, Phys. Rev. D36, 3291 (1987).

[7] J. P. Blaizot, E. Iancu, A. Rebhan, Phys. Lett. B523, 143-150 (2001).

[8] C. Ratti, R. Bellwied, M. Cristoforetti and M. Barbaro, Phys. Rev. D 85, 014004 (2012)

[9] V. Koch, A. Majumder and J. Randrup, Phys. Rev. Lett. 95, 182301 (2005)

[10] Z. Fodor and S. D. Katz, Phys. Lett. B 534 (2002) 87

[11] Z. Fodor and S. D. Katz, arXiv:0908.3341 [hep-ph].

[12] S. .Borsanyi, G. Endrodi, Z. Fodor, S. D. Katz, S. Krieg, C. Ratti and K. K. Szabo, arXiv:1204.6710 [hep-lat].

[13] S. Borsanyi, Z. Fodor, S. D. Katz, S. Krieg, C. Ratti and K. Szabo, JHEP 1201, 138 (2012)

[14] R. V. Gavai, S. Gupta, Phys. Rev. D73, 014004 (2006). M. Cheng et al., Phys. Rev. D79, 074505 (2009). A. Bazavov et al., Phys. Rev. D 80, 014504 (2009).

[15] Y. Aoki, Z. Fodor, S. D. Katz and K. K. Szabo, Phys. Lett. B 643, 46 (2006)

[16] Y. Aoki, S. Borsanyi, S. Durr, Z. Fodor, S. D. Katz, S. Krieg and K. K. Szabo, JHEP 0906, 088 (2009)

[17] S. Borsanyi et al. [Wuppertal-Budapest Collaboration], JHEP 1009, 073 (2010) 